\title{
Ambientes, relaciones e interacciones como un tejido sistémico en los momentos cotidianos de aeioTU
}

Volumen $6 \mathrm{~N} .{ }^{\circ} 46$ enero - junio de 2019

ISSN: 0122-4328

ISSN-E: 2619-6069

pp. $89-98$

\author{
Environments, \\ Relationships and \\ Interactions as a \\ Systemic Fabric in \\ the Daily Moments \\ of aeioTU
}

\author{
Ambientes, \\ relacionamentos \\ e interações como \\ tecido sistêmico \\ nos momentos \\ cotidianos da aeioTU
}

\section{Camila del Pilar Díaz Carranza* Nancy Rocío Vargas Galindo*}

Fecha de recepción: 1-10-18

Fecha de aprobación: 2-07-19

\section{PARA CITAR ESTE ARTÍCULO}

Díaz, C., y Vargas, N. (2019). Ambientes, relaciones e interacciones como un tejido sistémico en los momentos cotidianos de aeioTU. Nodos y nudos, 6(46), 13-26. doi: 10.17227/ nyn.vol6.num46-8638
* Licenciada en Educación Infantil de la Universidad Pedagógica Nacional, especialista en Educación y orientación familiar de la Fundación Universitaria Monserrate y estudiante de la Maestría en Familia, educación y desarrollo; maestra licenciada de aeioTU Pasadena. cami8807@hotmail.com

* Licenciada en Pedagogía Infantil de la Universidad Distrital Francisco José de Caldas y estudiante de la Especialización en Desarrollo Humano con énfasis en procesos afectivos y creatividad de la misma universidad. Miembro fundador del Colectivo Cucurumbé, organización que aborda la implementación de la Cátedra de Estudios Afrocolombianos y Maestra Licenciada de aeioTU Pasadena. narovaga@gmail.com. 


\section{RESUMEN}

Este texto es una reflexión sobre cómo en el epacio educativo institucional aeioTU Pasadena, fundación que promueve el potencial de la primera infancia inspirado en la filosofía de Reggio Emilia, se entretejen los ambientes físicos junto al ambiente social como un conjunto de elementos que interacúan entre sí, es decir, un sistema en el cual emergen conexiones constantes que son componentes indispensables para la consolidación de vinculos, la construcción de conocimientos y de relaciones consigo mismo y con los demás. De este modo, se promueven procesos de desarrollo integrales transformadores que trascienden los diferentes escenarios de quienes viven la experiencia educativa.

Volumen 6 N. ${ }^{\circ} 46$ enero - junio de 2019 ISSN: 0122-4328 ISSN-E: 2619-6069 pp. $89-98$
Palabras clave: enfoque sistémico; nutrición relacional; primera infancia

\section{ABSTRACT}

This text is a reflection on how in the institutional educational space aeioTU Pasadena, a foundation that promotes the potential of early childhood inspired by the philosophy of Reggio Emilia, the physical environments are interwoven together with the social environment as a set of elements that interact with each other, it means, a system in which constant connections emerge that are indispensable components for the consolidation of bonds, the construction of knowledge and relationships with oneself and with others. In this way, transformative integral development processes are promoted that transcend the different scenarios of those who live the educational experience.

Keywords: systemic approach; relational nutrition; early childhood

\section{RESUMO}

Este texto é uma reflexão sobre como no espaço educacional institucional aeioTU Pasadena, uma fundação que promove o potencial da primeira infância inspirada na filosofia de Reggio Emilia, os ambientes físicos se entrelaçam com o ambiente social como um conjunto de elementos que interagem entre si ou seja, um sistema no qual emergem conexões constantes, componentes indispensáveis para a consolidação de vínculos, a construção de conhecimentos e relações consigo mesmo e com os outros. Dessa forma, são promovidos processos de desenvolvimento integral transformador que transcendem os diferentes cenários daqueles que vivem a experiência educacional.

Palavras-chave: abordagem sistêmica; nutrição relacional; primeira infância 


\begin{abstract}
La escuela es, en realidad, un organismo vivo, un sistema. Decir que es un sistema significa poner el acento sobre el dinamismo y la constructividad de las relaciones e interacciones entre pares, de manera que cada parte actúa en el organismo como totalidad y viceversa.
\end{abstract}

LORIS MALAgUZZI

Se hace indispensable desde un enfoque sistémico elaborar una reflexión alrededor de las interacciones y posibilidades que genera todo un ambiente creado para promover una educación de calidad en la primera infancia. Este enfoque se piensa como nuevo paradigma que aborda las realidades de manera compleja por medio de un principio de base de distintas disciplinas enfocadas en este caso en lo educativo.

La reflexión pedagógica se da dentro de un contexto en el que el modelo educativo aeioTU está inspirado en la filosofía de Reggio Emilia y es la base de las experiencias y fundamentos pedagógicos de los cuales se impregnan las relaciones e interacciones que se tejen con la comunidad, las familias, los docentes, los niños y todo el equipo educativo. En este entorno se genera el ser conscientes de la confluencia de todos los seres y se reflexiona sobre las emociones, el pensar y el actuar en la relación consigo mismo y con los otros en la cotidianidad de los ambientes de aprendizaje.

Y para dar convergencia a todos los aspectos de desarrollo integral de la primera infancia, se piensan todas las relaciones sociales, aquellos vínculos afectivos que van más allá de las palabras descriptivas pero que a su vez son posibles de narrar desde la experiencia y el sentir como un tejido que se compacta, como conexiones que se van acercando más y más, como un convivir desde el aprender a comprender y conocer al otro, con lo otro y conmigo. Desde este enfoque sistémico es que se hace posible una educación con sentido ético, pedagógico y emocional con los niños.

\section{¿Qué es la filosofía de Reggio Emilia en aeioTU?}

Loris Malaguzzi, en 1946, luego de la Segunda Guerra Mundial, acompañado de familias y toda una comunidad en Italia, empezó a construir un concepto de infancia distinto que se concretó en la creación de una escuela que transformara la sociedad y brindara posibilidades enormes a los niños y las niñas.

Desde entonces todo un proceso de estudio alrededor de la educación en los primeros años parte de reconocer el potencial de los niños y edifica otra imagen de la infancia encaminada a reconocer su aporte para la transformación de la sociedad. En el aeioTU acogemos aspectos indispensables de Reggio Emilia como lo es reconocer a la infancia en el marco de sus derechos fundamentales, siendo la educación inicial uno de ellos, por tal razón esta debe ser coherente a su desarrollo integral, sus necesidades e intereses.

En esa misma línea, se concibe el aprendizaje como un proceso en el que el niño es protagonista y co-constructor en la cotidianidad; en cada momento se generan aprendizajes y hasta el error es valioso para Malaguzzi. El niño está inmerso en un flujo de saberes y relaciones que nutren así las diversas formas de aprender, de relacionarse y de construir con el otro, por lo que para el aeioTu es valioso el valor de los diversos lenguajes de los niños.

Esto implica reconocer las diferencias, la diversidad de formas de ver el mundo y las posibilidades de ser, de estar y de comprenderlo para habitarlo, mientras se entablan interacciones que de la mano con los ambientes y los espacios diseñados para la infancia, se de una confluencia de saberes que permitan una educación integral.

Según la filosofía de Reggio Emilia, un centro de atención es un nido y se describe como: "un sistema de comunicación integrado en el sistema social más amplio: un sistema de comunicación, de socialización, de interacciones en el que hay tres principales interesados afectados por el proyecto educativo, es decir, el niño, el educador y la familia" (Rinaldi, 2006, p. 27).

Según lo anterior, nuestra institución se posiciona en una visión holística de las realidades en las que nos encontramos, en este nido, en el cual el bienestar de todos es fundamental para forjar una educación de calidad que involucre los ambientes físicos y sociales, las relaciones e interacciones entre ellos y así un sistema social más amplio. 


\section{De los ambientes físicos a los vínculos afectivos}

Los espacios y ambientes en aeioTU están diseñados y pensados como espacios de aprendizaje, conexiones, acogimiento, "dispuestos de una manera tal que posibilitan e invitan al juego, la exploración, el asombro y la investigación. Así el ambiente y los espacios se modifican para tomar forma a medida que los procesos o proyectos de aprendizaje se van configurando y se llevan a cabo con los niños, de este modo cobran sentido y adquieren valor gracias a ellos" (Fundación Carulla aeioTU, 2015, p. 49).

Habitar un espacio significa entonces que este se va permeando de la identidad de quienes están en él: todo un equipo de trabajo conformado por los educadores, los colaboradores, las familias, los maestros y los niños, quienes se hacen visibles y ocupan un lugar preponderante. Y ello se genera a partir de un proceso de intercambio de saberes, de experiencias, de conocimiento del otro, de escucha activa de cada habitante y de su contexto, como una forma de reconocer las posibilidades y las necesidades del territorio, lo natural y lo artificial, las conexiones existentes entre ellos, qué nos aporta y qué podemos aportar.

Se van entonces configurando ambientes tranquilos, agradables, pedagógicos, estéticamente pensados y "el cuidado que se le da a este permite que se construya una relación de cuidado, pertenencia, familiaridad y seguridad, propicias para el diálogo y la reflexión" (Fundación Carulla aeioTU, 2015, p. 49). Ejemplos de esta experiencia pueden ser llegar y ver un pequeño espacio lleno de cojines, luces, un enorme tapete suave y diferentes cuentos que provocan llegar a leer o un comedor con disposiciones de alimentos en recipientes o llegar al aula y encontrar varios rincones con provocaciones diversas, lo cual implica iniciar el día con la expectativa y el agrado de compartir las exploraciones de cada lugar.

Estos espacios permiten, como lo menciona Alfredo Hoyuelos (2005), dividirse y subdividirse para que los niños puedan encontrarse naturalmente en pequeños grupos o en la intimidad si lo desean (p. 2), para desde alli empezar a generar posibilidades en los ambientes físicos que den lugar a interacciones amorosas, cercanas, que creen conexiones y construyan aprendizajes, relaciones e interacciones.

El proyecto de Reggio Emilia configura además unos factores importantes para el diseño de ambientes como se menciona en el apartado siguiente:

Osmosis (integración de la escuela en el contexto próximo), habitabilidad (escuelas acogedoras donde el espacio contiene y da tiempo), identidad (la escuela creadora de sentido de pertenencia y como lugar para la transición, la calma y el encuentro), relación (una escuela capaz de generar calidad y calidez en las relaciones, encuentros e intercambios en una idea de transparencia: democracia y circularidad, etc.), constructividad (una escuela que diversifica los espacios para ofrecer posibilidades de juego simbólico y pre-simbólico, para la exploración, los recorridos múltiples, los escenarios de juego, los espacios polivalentes y de acontecimiento, espacios de retos, conquistas y desafios, espacios para sostener el deseo y la curiosidad, etc.), la polisensorialidad (una concepción del espacio con ambientes variados y equilibrados desde el punto de vista sensorial y perceptivo para crear diversos paisajes en la escuela: paisaje cromático equilibrado y no saturado de color y luz, los ambientes sonoros de la escuela, los espacios de olores, los paisajes matéricos, etc). Epigénesis (el espacio se transforma y se adapta a los proyectos de los niños y de los adultos, donde cada modificación promueve nuevas acciones y aprendizajes a partir de estructuras móviles con posibilidades de transformación, construcción-deconstrucción, escenarios de juego cambiantes, etc). Narración (el espacio como memoria de la escuela depositada en objetos-memoria e imágenes para la documentación y la información visual en fotografías y paneles). (Abad, 2008, p. 4).

Es de esta manera que disponiendo el mobiliario a la altura de los niños, y materiales y provocaciones pensadas para la diversidad de la infancia es que confluye en el aula un ambiente polisensorial que invita a la confluencia o la individualidad, y en donde se manifiestan las construcciones y saberes elaborados por los niños con antelación, pero que a su vez les permite construir otros saberes.

Así, se da la conformación de una escuela transformadora que se va tejiendo, forjada por las relaciones y vínculos que permiten jugar con el otro, esconderse y aparecer, compartir un descubrimiento, leer las posibilidades que se puedan crear, de modo que se represente en ese espacio la identidad e intereses de todo 
un equipo. De esta manera, Cabanellas y Eslava (2005) reconocen que este es:

Una elección consciente de espacios, formas relacionales, vacíos, llenos, materiales, texturas, colores, luces, sombras, olores, etc., que deben potenciar, ayudar y reflejar la convivencia pedagógica y cultural que se construye en las instituciones educativas, creando vinculos que hagan posible la definición de las diversas identidades. (p. 70).

El niño tiene derecho entonces en su educación inicial a participar de espacios de calidad, cálidos, organizados y con propuestas en pro de su aprendizaje, donde se promueve la creatividad y la resolución de problemas, donde se investiga y se genera conocimiento con los otros; no solo se piensa el espacios físico, sino todas las posibilidades que en él se pueden crear.

Asimismo, Malaguzzi (citado por Cabanellas y Eslava, 2005) plantea el trabajo en grupos pequeños como estrategia para compartir y relacionarse en los ambientes propuestos, pues menciona que los niños naturalmente se unen y pueden dialogar y discernir con el otro; el trabajo en grupos pequeños lo plantea desde la pedagogía relacional, en la que el conversar y la comunicación en diferentes lenguajes son aspectos fundamentales para aprender con los demás, y además se fortalecen en la medida que el grupo continúe las investigaciones o exploraciones realizadas.

Es así como un ambiente físico se concibe en aeioTU como tercer maestro por la posibilidad de aprendizajes que se pueden provocar desde alli, pero además movilizando vínculos afectivos indispensables para una escuela que trascienda e impacte toda una sociedad.

\section{De las relaciones e interacciones sociales como procesos de nutrición relacional}

En el centro aeioTu Pasadena, un espacio en el que transita la infancia desde sus primeros años de vida, se generan relaciones, interacciones y conocimientos constantemente en cada uno de los momentos del día con todas las personas que están allí, y se generan oportunidades de aprendizaje especialmente en la socialización y el desarrollo socioafectivo, los cuales son determinantes para encaminar otros procesos.
En este sentido, las maestras, el equipo de apoyo (la coordinadora, la parte administrativa, la psicóloga, la nutricionista y el tallerista), caseras, manos amigas y el conserje generan diálogos en los que hay una relación directa, participativa, consciente y de escucha, en los cuales se reconoce social, cultural y políticamente a los niños, valorando sus saberes, inquietudes, ideas, hipótesis y sentires que se construyen en la cotidianidad.

Así, desde el ingreso, el conserje con la bienvenida reconoce a los niños y a las familias, escuchando las historias que encuentran de camino al centro, de las novedades de la huerta, el juego y la imaginación que permite el andar diario; el equipo de apoyo con su alegría, acercamiento y preguntas, promueven los diálogos y la confianza, las caseras con su preocupación por el cuidado en la alimentación se encuentran atentas al gusto de los niños y a las percepciones alrededor de cada plato de comida y las maestras siempre acompañan todos los procesos en los que la autonomía, el respeto y la validación son esenciales en cada momento.

De este modo, el equipo del centro, maestras y familias son actores nutricios en la conformación de la subjetividad de los niños, pero a la vez se involucran en la transformación de saberes y en la configuración de las identidades.

La nutrición relacional como parte de un análisis desde un enfoque sistémico permite así describir y puntualizar el concepto asignado por el psiquiatra, psicólogo, doctor en medicina y terapeuta sistémico español Linares (2012), quien denomina la nutrición relacional como aquellos aspectos emocionales, cognitivos y pragmáticos que constituyen el amor, y lo denomina complejo por la variedad de formas que lo conforman. Este autor configura todo un sistema de relaciones basadas en el amor que conforman la personalidad de cada sujeto desde la vivencia subjetiva de ser amado (p. 28).

El niño desde que nace mantiene relaciones con sus padres o cuidadores en el que ese amor se hace evidente desde un pensar, sentir y hacer amoroso, que se configura en procesos relacionalmente nutricios para la formación y el crecimiento de cada ser humano; por tanto, es impresindible pensar la concepción de los niños, ya que implica una responsabilidad y respeto hacia el otro, dar y recibir en las interacciones cotidianas para construirnos y co-construirse. 


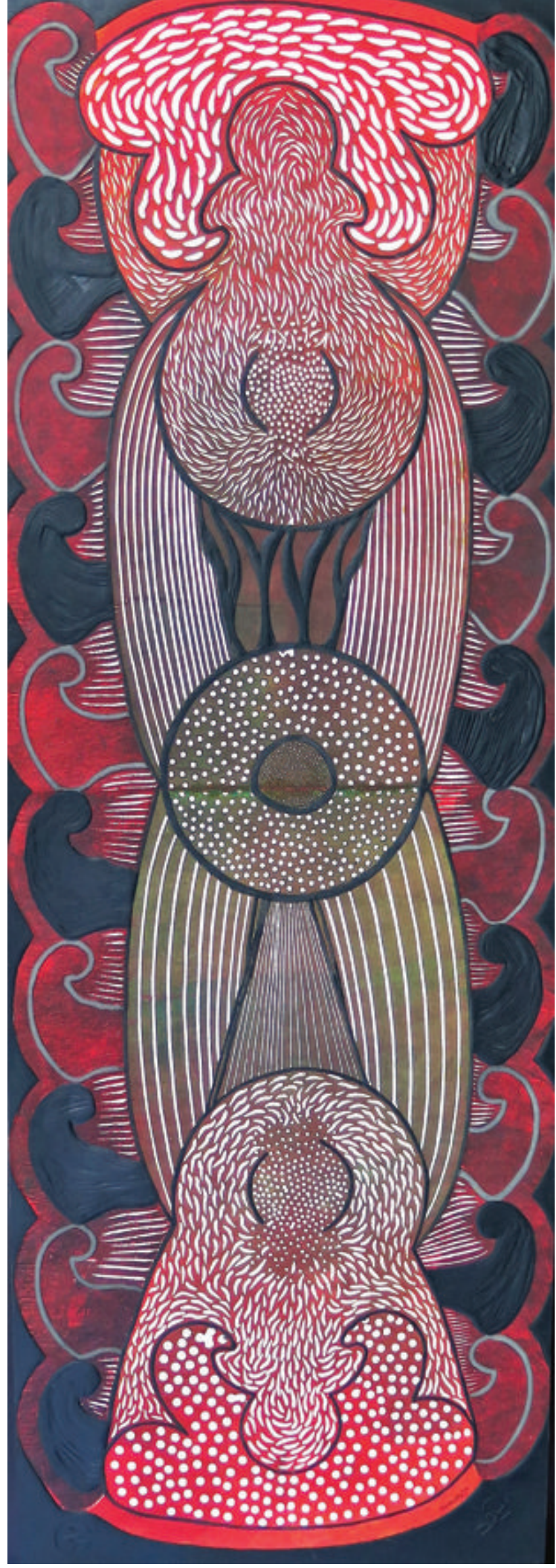


En relación con los componentes pragmáticos (acciones) de los vínculos parento-filiales se encuentra la socialización de la que se derivan conceptos de protección y normatividad, los cuales se refieren a las acciones reales que los padres ejercen con sus hijos para conformar relaciones nutricias; sin embargo, este aspecto se articula al ámbito educativo en tanto los maestros también ejercen acciones encaminadas a la construcción de relaciones nutricias especialmente en la primera infancia.

Además, otro aspecto que plantea Linares (2012) es "aquella necesidad de verse como un individuo con capacidades y características propias, validado desde su sentir, necesidades y habilidades." (p. 30), lo cual genera también procesos de nutrición que consolida subjetividades.

Las relaciones nutricias, entonces, pueden ser compensadas por otras personas o situaciones distintas al núcleo familiar, se consolidan en primera infancia también por los maestros y todo un equipo educativo cómo se vive en aeioTU al formar parte de las vivencias y brindar relaciones amorosas. A su $v e z, y$, en cualquier caso, "una personalidad madura no puede construirse sin los aportes emocionales de la nutrición relacional, que son el cariño y la ternura" (Linares, 2000, p. 57); es decir, esos componentes relacionales que corresponden a las manifestaciones afectivas $y$, además, a las prácticas de cuidado y amor, así como a las percepciones de los niños y de equipo frente a ello, lo cual se ve reflejado en el deseo por encontrarse alli de los infantes, las manifestaciones afectuosas constantes con cada persona del equipo de trabajo y las relaciones cercanas con muchas de las familias.

Maturana (2001) afirma que es el amor la emoción que posibilita el lenguaje, la emoción central en la historia evolutiva humana, la emoción que constituye el dominio de acciones en que nuestras interacciones recurrentes legitiman el otro en la convivencia, es decir, se rescata la importancia de las emociones en medio de un pensamiento constituido por paradigmas en los que la racionalidad tiene una valía superior y las emociones quizá son la simple expresión de un ser humano no pensante.
No encontramos frente al dilema del propósito individual versus el propósito social, pues la necesidad de ser competitivo genera una negación del otro y, por ende, una pérdida del tejido social. En este sentido, se reconoce el educar como un proceso de transformación en la convivencia con otro, en ese espacio compartido, en esas relaciones de aceptación e interacción reciproca en el que es indispensable la reflexión; estos planteamientos difieren con una educación que estimula la competencia, la apropiación y la explotación del mundo natural sobre de la coexistencia y la aceptación legítima del otro.

Es entonces el amor, las relaciones y las emociones las que permiten proveer un ambiente nutricio para la primera infancia, en el que se hace un aporte importante a la construcción de las subjetividades de los sujetos inmersos en una sociedad, de modo que se valide la primera infancia como un enfoque de trabajo indispensable y como un compromiso en la transformación de la sociedad.

\section{De las interacciones entre la familia y el equipo educativo}

La educación infantil en reggio Emilia se basa en una pedagogía relacional que sustenta el valor de las interacciones entre cada uno de los miembros del equipo desde un enfoque sistémico entendiéndolo como "una conjunción de elementos en interacción dinámica que tienen una finalidad común" (Malaguzzi, 2001, p. 55), lo que significa la inclusión de todos los participantes el el objetivo educativo de transformación e innovación.

Esto implica todo un trabajo de reconocer y conocer al otro, de saber los sueños, los caminos y los retos de cada ser en su mundo, para de esta manera acoger y validar al otro desde la postura de Maturana, mientras se construye una ruta conjunta de la que todos hacemos parte, aportamos y, día a día, nos reconocemos desde cada rol, desde cada construcción subjetiva del mundo; es necesario, como dice Malaguzzi (2001), encontrar una alianza con las cosas y con la organización del trabajo (p. 62). 
Las relaciones con el conserje, las caseras, las manos amigas, las maestras, la sección administrativa y de coordinación, en la medida en que se tejen desde la confianza, el compromiso y el amor, pero además desde el saber pedagógico, el valor por el potencial de los niños y sus capacidades, la disciplina positiva, la construcción de saberes colectivos entre el equipo como riqueza natural además de la consciencia de la consolidación de sociedad son puntos claves para emprender un objetivo educativo común en la primera infancia.

Ligado a ello, se suma el ser un centro de puertas abiertas con la posibilidad de generar encuentros cada mañana con las familias, y en el que se trabaja para el conocimiento y el reconocimiento de cada una de ellas, se trata de entablar las interacciones necesarias para movilizar a todo un sistema (niño) en interacción con otros (familia y escuela), pero, además, dentro de uno mayor: el contexto sociocultural.

Las familias participan de manera activa en nuestra cotidianidad, se van contagiando del ambiente, de la acogida que se percibe y así van formando parte de la construcción de saberes, comparten algunas habilidades específicas, como al compartir lo que hacen en familia con todo un grupo o participar en una experiencia que hace parte de los proyectos de investigación de cada uno, y, de esta manera, aportan en gran medida a los aprendizajes que los niños asumen con mayor asombro y alegría al sentir conexión con cada ámbito de sus vidas.

En esta construcción cada una de las partes se mueve con su propia identidad y sus deberes, interrelacionando vínculos y confrontaciones reciprocas. $Y$ esto hace que el sistema sea abierto y tenga una trama que asocia las partes y el todo y posea una capacidad autorreguladora y de acomodación, también, con respecto a los acontecimientos que son imprevistos y aleatorios. (Malaguzzi, 2001, p. 61).

Esto implica un alto compromiso con la labor educativa en tanto que se involucra a las familias como actores activos en los procesos de aprendizaje y el desarrollo de los niños, y además son fundamentales en la construcción de sujetos con experiencias significativas desde la primera infancia.

\section{De la ecología profunda a la construcción de proyectos de investigación que promueven interacciones dentro de un sistema-aula}

Dar una mirada general a los proyectos de investigación en cada una de las aulas alrededor de nuestro proyecto de Centro como "medio ambiente" permite una interacción entre pares, maestros, familias y articuladores culturales, pero además una exteriorización al involucrar el ámbito ecológico: el macrosistema natural y artificial en el que nos encontramos inmersos y entablamos relaciones diarias; es importante sentirnos parte de él para tejer relaciones de cuidado y sostenibilidad.

\section{Como menciona Malaguzzi,}

El niño aprende interaccionando con su ambiente, transformando activamente sus relaciones con el mundo de los adultos, de las cosas, de los acontecimientos y, de manera original, de los coetáneos. En este sentido participa en la construcción de su yo y en la construcción del de los otros. (Malaguzzi, 2001, p. 58).

Es indispensable desde un pensamiento sistémico entablar esas relaciones con el ambiente, en el que cada aula y proyecto de investigación que surge genera una riqueza de saberes que se comparten.

En caminadores el movimiento y las huellas que se deja al andar hacen parte de una exploración sensorial del mundo desde el interés y potencial de los niños entre 1 y 2 años; en exploradores (niños de 2 a 3 años) las casas, su construcción, la indagación constante por su estructura y conocer la de cada compañero, plantea el desarrollo constante de saberes a partir de un ambiente artificial en donde además se promueven las salidas alrededor del barrio para reconocer formas y conexiones con el ambiente; en aventureros (de 3 a 4 años) se realiza toda una indagación alrededor de la pregunta ¿de dónde nacen las frutas? Es toda una conexión con los alimentos pero además es un proyecto para la construcción de una huerta que permite establecer conexiones cercanas y conscientes con el medio ambiente; y en Investigadores (de 4 a 5 años), la reflexión en torno a la importancia de lavarse las manos 
enmarca toda una búsqueda por descubrir el mundo de los microorganismos y, de esta manera, se generan las conexiones con la conciencia del cuidado propio, pero también el de nuestro planeta.

Teniendo en consideración los planteamientos de Capra (1996), quien propone el concepto de la ecología profunda, se reconoce la interdependencia fundamental entre todos los fenómenos y el hecho de que, como individuos y como sociedades, estamos todos inmersos en los procesos cíclicos de la naturaleza (p. 28); lo que implica todo un tejido de relaciones que generan conexiones indispensables para promover en la primera infancia una educación que impacte en la sociedad y transformen las relaciones de consumo y la extracción de los recursos naturales desde una concepción desarrollista.

En esta línea, Serge Latouche (2004) plantea la dicotomía entre la visión mítica de ver el desarrollo como la realización de los deseos en beneficio de todos en contradicción con la realidad que en palabras textuales dice "es una empresa que pretende transformar en mercancía la relación de los hombres con la naturaleza" (p. 65).

Por otra parte, se promueve una línea que involucra lo ecológico a partir de los postulados de Renán Vega (2012), quien afirma que pretender que la vida humana es posible sin los ecosistemas, tal y como sostienen ciertos economistas y tecnócratas, no pasa de ser una falacia justificatoria del irracional modelo de acumulación capitalista.

Esto implica generar conexiones y con ello, como dice Leonardo Boff (citado en Sánchez, 2013) desarrollar una actitud de sentir con cuidado que debe transformarse en cultura y que exige un proceso pedagógico más allá de la escuela formal que penetre las instituciones y haga surgir un nuevo estado de conciencia y de conexión con la Tierra y con todo lo que existe y vive en ella.

Por ello la importancia de mantener una relación con el ambiente, con la naturaleza desde proyectos de investigación acordes con los intereses y necesidades de aquel nido y aula en los que emergen conexiones importantes que se ven reflejadas en las siguientes ideas de los niños:
» "Los ladrillos son de 1, 2, 3, 4 líneas" (Emilio, 3 años).

» "Las fresas tienen semillas y estan a los bordes" (Julieta, 3 años).

» "Ahí hay gérmenes, en esa agua sucia del piso, es un charco sucio, porque está negrita y cerca del caño" (Daniel Espitia, 5 años; recorrido por el barrio Alhambra).

\section{Reflexión final}

Desde un enfoque sistémico en el que las partes se relacionan con el todo y el todo con las partes, es indispensable pensarnos en una transformación desde las relaciones nutricias fundamentadas en el amor, en brindar ambientes de calidad para forjar una educación inicial que de paso a seres humanos para sí mismos, para los otros y para un mundo que es de todos.

Por lo cual, el ambiente social, físico y emocional confluyen en la reflexión y práctica pedagógica como una apuesta por pensar en una educación inicial con sentido, para la vida y para la cotidianidad de los niños, siendo intencionada y en una relación horizontal en la que se construye el ser humano en espacios tranquilos y potenciadores.

\section{Referencias}

Abad, J. (2008). La escuela como ámbito estético según la pedagogía reggiana. Recuperado de https://www.vitoria-gasteiz.org/wb021/http/contenidosEstaticos/adjuntos/es/33/07/43307.pdf

Boff, L. (2002). Ecología: grito de la tierra, grito de los pobres

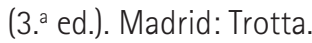

Cabanellas, I. y Eslava, C. (2005). Territorios de la infancia. Diálogos entre arquitectura y pedagogía. Barcelona: Graó.

Capra, F. (1996). La trama de la vida. Una nueva perspectiva de los sistemas vivos. Barcelona: Editorial Anagrama.

Fundación Carulla aeioTU. (2015). Libro Cartografía curricular. Horizontes, orientaciones pedagógicas y operativas para la implementación de la experiencia educativa aeioTU. Bogotá, Colombia.

Hoyuelos, A, (2005). La cualidad del espacio ambiente en la obra pedagógica de Loris Malagozzi. Barcelona: Graó.

Latouche, S. (2004). Sobrevivir al desarrollo: de la descolonización del imaginario económico a la construcción de una sociedad alternativa. Barcelona, España: Icaria Editorial. 
Linares, J. (2012) Terapia Familiar Ultramoderna. Barcelona: Editorial Herder.

Linares, J. y Campo, C. (2000). Tras la honorable Fachada. Los trastornos depresivos desde una perspectiva relacional. Barcelona: ediciones Paidos Ibérica S.A.

Malaguzzi, L. (2001). La educación infantil en Reggio Emilia (Trad. de Alfredo Hoyuelos). Barcelona: Octaedro.

Maturana, H. (2001). Emociones y Lenguaje en educación y politica. Santiago de Chile: Dolmen Ensayo.

Maturana, H. y Porksen, B. (2004). Del ser al Hacer, los orígenes de la biología del conocer. Santiago de Chile: JC Saez editor.
Sanchez, T. (2013). El cuidado esencial, una propuesta ética de actualidad. Revista Quaestiones Disputatae, 12. Recuperado de http://revistas.ustatunja.edu.co/index.php/ qdisputatae/article/view/261

Rinaldi, C. (2006) In Dialogue with Reggio Emillia, listening, researching and learning (Contesting Early Childhood). Series Editors: Gunilla Dahlberg and Peter Moss.

Vega, R. (2012). El imperialismo ecológico. El interminable saqueo de la naturaleza y de los parias del sur del mundo. Revista Herramienta, 62. Recuperado de https://herramienta.com.ar/articulo.php?id=341

\section{Diálogo con el conocimiento}

Desde una mirada estética, el ambiente es el lenguaje que comunica a quienes lo habitan desde unas maneras de ser, de estar y de relacionarse con el entorno, los objetos, los otros y consigo mismo. En una institución, el diseño de ambientes refleja una concepción de infancia, de maestro y de educación: la manera como se organizan y disponen los materiales y recursos puede limitar o potenciar el juego, el trabajo pedagógico alrededor del cuerpo y el movimiento, el arte con todas sus manifestaciones, la literatura y la exploración del medio, los cuales constituyen los pilares del armazón pedagógico de la educación infantil. En tal sentido, el ambiente es una ventana de oportunidades para que quienes lo habitan se sientan acogidos, contenidos, albergados, reconocidos y valorados; un ambiente es la posibilidad de reconocer las diferencias, tejer vínculos, relaciones e interacciones.

Por último, será pertinente añadir que diseñar ambientes es en la actualidad uno de los principales desafios de los maestros, porque implica pensar en las preguntas históricas que se hace un profesor en el marco de un acto pedagógico y que están articuladas al qué, el para qué y el cómo enseñar y provocar experiencias que potencien el desarrollo en los niños de primera infancia.

Sandra Marcela Durán Chiappe Licenciatura en Educación Infantil Departamento de Psicopedagogia Facultad de Educación Universidad Pedagógica Nacional smduran@pedagogica.edu.co 